\title{
Compromized geranylgeranylation of RhoA and Rac1 in mevalonate kinase deficiency
}

\author{
L. Henneman • M. S. Schneiders • M. Turkenburg • \\ H. R. Waterham
}

Received: 9 March 2010 /Revised: 25 May 2010/Accepted: 7 July 2010 /Published online: 3 September 2010

(C) The Author(s) 2010. This article is published with open access at Springerlink.com

\begin{abstract}
Mevalonate kinase deficiency (MKD) is an autoinflammatory disorder caused by mutations in the $M V K$ gene resulting in decreased activity of the enzyme mevalonate kinase (MK). Although MK is required for biosynthesis of all isoprenoids, in MKD, in particular, the timely synthesis of geranylgeranyl pyrophosphate appears to be compromised. Because small guanosine triphosphatases (GTPases) depend on geranylgeranylation for their proper signaling function, we studied the effect of MK deficiency on geranylgeranylation and activation of the two small GTPases, RhoA and Rac1. We demonstrate that both geranylgeranylation and activation of the two GTPases are more easily disturbed in MKD cells than in control cells when the flux though the isoprenoid biosynthesis pathway is suppressed by low concentrations of simvastatin. The limited capacity of geranylgeranylation in MKD cells readily leads to markedly increased levels of nonisoprenylated and activated GTPases, which will affect proper signaling by these GTPases.
\end{abstract}

$\begin{array}{ll}\text { Abbreviations } \\ \text { MKD } & \text { Mevalonate kinase deficiency } \\ \text { MK } & \text { Mevalonate kinase }\end{array}$

Communicated by: K. Michael Gibson

References to electronic databases: mevalonate kinase deficiency: OMIM \#260920, \#610377. Gene map locus: 12q24. Gene symbol: MVK. Mevalonate kinase: EC 2.7.1.36.

Competing interest: None declared.

L. Henneman • M. S. Schneiders • M. Turkenburg •

H. R. Waterham $(\triangle)$

Laboratory Genetic Metabolic Diseases (F0-222),

Departments of Clinical Chemistry and Pediatrics,

Academic Medical Center, University of Amsterdam,

P.O. Box 22700, 1100 DE Amsterdam, The Netherlands

e-mail: h.r.waterham@amc.uva.nl
Hyper-IgD Hyperimmunoglobulinemia D and periodic fever syndrome

GEF Guanine nucleotide exchange factor

GAP GTPase-activating protein

GDI GDP-dissociation inhibitor

GGTI Geranylgeranyltransferase inhibitor

\section{Introduction}

Protein isoprenylation is the posttranslational covalent addition of the isoprenoids farnesyl pyrophosphate or geranylgeranyl pyrophosphate to cysteine residues at the carboxy terminus of proteins (Casey and Seabra 1996). Isoprenylation is crucial for the proper function of small guanosine triphosphatases (GTPases) (McTaggart 2006; Takai et al 2001), because it enables their localization to membranes where they can interact with downstream signalling effectors. Small GTPases participate in the regulation of a wide variety of cellular functions, including cell cycle progression, morphology and migration, cytoskeletal function, vesicle trafficking, and gene transcription (Casey and Seabra 1996; McTaggart 2006; Takai et al 2001). In addition to regulation by isoprenylation, small GTPases act as molecular switches by cycling between an active and an inactive guanosine diphosphate (GDP)-bound state (Bustelo et al 2007). Activation of small GTPases is controlled by so-called guanine nucleotide exchange factors (GEFs) that catalyse the exchange of GDP for GTP, whereas GTPase-activating proteins (GAPs) release the interaction with downstream effectors by accelerating hydrolysis of GTP by the small GTPases. Cytosolic RhoGDP-dissociation inhibitors (Rho-GDIs) normally prevent 
activation of small GTPases in the cytosol through binding with their geranylgeranyl moieties (Bustelo et al 2007).

Among the different enzyme defects of isoprenoid biosynthesis currently known, only the autoinflammatory disorder mevalonate kinase deficiency (MKD; OMIM \#260920, \#610377) affects the synthesis of all isoprenoids (Waterham 2006). MKD is due to mutations in the $M V K$ gene, resulting in decreased activities of mevalonate kinase (MK; EC 2.7.1.36), the first enzyme following the highly regulated 3-hydroxy-3-methylgluaryl (HMG-CoA reductase) in the isoprenoid biosynthesis pathway (Houten et al 2003a; Waterham 2006). Dependent on the mutations, patients may present with the milder hyperimmunoglobulinemia D and periodic fever (Hyper-IgD)-syndrome phenotype characterized primarily by recurrent episodes of high fever associated with headache, skin rash, abdominal pain, arthritis, nausea, and diarrhoea or with the more severe mevalonic aciduria phenotype, where these episodes are accompanied with developmental delay, dysmorphic features, ataxia, cerebellar atrophy, and psychomotor retardation (Houten et al 2003a).

Despite the markedly decreased MK enzyme activity in fibroblasts from MKD patients, the de novo isoprenoid biosynthesis can be rather normal when these fibroblasts are cultured under normal conditions (Gibson et al 1990; Hoffmann et al 1997; Houten et al 2003b). Previous work showed that this is due to an increased activity of HMGCoA reductase, which leads to elevated levels of mevalonate and a virtually normal flux through the isoprenoid biosynthesis pathway (Gibson et al 1990; Hoffmann et al 1997; Houten et al 2003b). However, this flux can easily be disturbed, which also follows from the observation that MKD fibroblasts are more sensitive to inhibition of HMGCoA reductase by simvastatin than are control fibroblasts (Houten et al 2003b). Although the depressed MK enzyme activity in MKD in principle affects the biosynthesis of all isoprenoids, there are strong indications that, in particular, a temporary shortage or dysfunction of one or more geranylgeranylated proteins is associated with onset of fever episodes (Frenkel et al 2002; Houten et al 2002; Houten et al 2003b; Mandey et al 2006).

Because small GTPases depend on isoprenylation for their proper signalling function, we studied the effect of MK deficiency on isoprenylation (i.e., geranylgeranylation) and activation of the two small GTPases, RhoA and Rac1. RhoA and Rac1 are two major examples of the family of RhoA GTPases that play a role in different cellular processes (including inflammatory processes), are abundantly expressed, and for which good antibodies and sensitive activation assessment assays are available. For these studies, we used cultured control and MKD fibroblasts in which the isoprenoid biosynthesis pathway can be readily manipulated by varying the culturing conditions.

\section{Materials and methods}

\section{Cell culture}

Primary skin fibroblast cell lines obtained from an MKdeficient patient with the classic mevalonic aciduria presentation and homozygous for the c.803T $>C$ (p.I268T) mutation in the $M V K$ gene, and a healthy control individual with similar passage numbers, were cultured in nutrient mixture of Ham's F-10 with L-glutamine and $25 \mathrm{mM} \mathrm{4-2-}$ hydroxyethyl-1-piperazineethanesulfonic acid (HEPES; Invitrogen, Breda, The Netherlands) supplemented with $10 \%$ fetal calf serum (FCS; Invitrogen) and grown in a temperature- and humidity-controlled incubator $[95 \%$ air, $5 \%$ carbon dioxide $\left.\left(\mathrm{CO}_{2}\right)\right]$ at $37^{\circ} \mathrm{C}$ until confluence. Subsequently, the medium was replaced with fresh culture medium supplemented with either $20 \mu \mathrm{M}$ geranylgeranyltransferase inhibitor (GGTI-298; Calbiochem, Merk Chemicals Ltd, Nottingham, UK) or different concentrations of simvastatin (a gift from Merck, Sharpe, and Dohme BV, Haarlem, The Netherlands), as indicated. Cells were then cultured for 2 days at $37^{\circ} \mathrm{C}$ and either used directly for the activated RhoA and Rac1 assessment assays (see below) or harvested by trypsinization, washed once with phosphatebuffered saline (PBS) and once with $0.9 \%$ sodium chloride $(\mathrm{NaCl})$, snap-frozen as pellets in liquid nitrogen, and stored at $-80^{\circ} \mathrm{C}$ to be used later for membrane and soluble fraction separation (see below). Simvastatin was prepared as a 10$\mathrm{mM}$ stock solution, as described previously (Houten et al 2003b). GGTI was prepared as a $20-\mathrm{mM}$ stock solution by dissolving the drug in dimethylsulfoxide (DMSO) (Merck, Darmstadt, Germany).

\section{Membrane and soluble fraction separation}

Membrane and soluble fraction separation was performed essentially as described previously (Houten et al 2003b) and used to determine levels of isoprenylated protein (i.e., membrane-bound) and nonisoprenylated protein (i.e., soluble fraction). Protein concentration in the sonicated lysates was determined using the Bradford assay (Biorad) and adjusted with hypotonic buffer to a concentration of $1 \mathrm{mg} /$ $\mathrm{ml}$. One milligram of total protein was used for ultracentrifugation. The membrane and soluble fractions were analyzed by immunoblot analysis, as described below. These experiments were performed in triplicate.

Activated RhoA and Rac1 assessment assays

Activated RhoA and Rac1 assessment assays were performed as described previously (Sander et al 1998). Briefly, cultured fibroblasts were washed three times with ice-cold PBS and subsequently lysed by scraping in the culture flask 
using lysis buffer [50 mM Tris pH 7.4, $100 \mathrm{mM} \mathrm{NaCl}, 10 \%$ glycerol, 1\% tergitol-type NP-40 (NP-40), 2 mM magnesium chloride $\left(\mathrm{MgCl}_{2}\right), 0.1 \mathrm{mM}$ phenylmethylsulfonyl fluoride, $10 \mu \mathrm{g} / \mathrm{ml}$ leupeptin, $10 \mu \mathrm{g} / \mathrm{ml}$ aprotinin, $1 \mathrm{mM}$ benzamidine, $1 \mathrm{mM}$ dithiothreitol (DTT), $1 \mathrm{mM}$ vanadate]. The lysed cell homogenates were then centrifuged (10 min, $12,000 \times \mathrm{g}$ ) and the supernatants transferred to $1.5-\mathrm{ml}$ tubes. After determining protein concentration of the supernatants with the Bradford assay, $500 \mu \mathrm{g}$ total protein in $500 \mathrm{ml}$ was incubated for $60 \mathrm{~min}$ at $4^{\circ} \mathrm{C}$ with bacterially produced glutathione $S$-transferase Ras-binding domain (GST-RBD, Rhotekin) (Reid et al 1996) (for RhoA pulldowns) or GST-p21-activated Ser/Thr kinase (PAK) (Sander et al 1998) (for Rac1 pulldowns) bound to glutathione-agarose beads (Sigma, St. Louis, MO, USA). Subsequently, the beads were washed three times with lysis buffer followed by centrifugation (10 s, 12,000 x $g$ ). Bound proteins, i.e., active RhoA or Rac1, were eluted by boiling in sodium dodecyl sulfate (SDS)-sample buffer and analyzed by immunoblot analysis, as described below. These experiments were performed in triplicate.

Combination of membrane and soluble fraction separation and activated RhoA and Racl assessment assays

For combination assays, fibroblasts were incubated with 0 , 0.2 , or $0.02 \mu \mathrm{M}$ simvastatin for $48 \mathrm{~h}$ at $37^{\circ} \mathrm{C}$. Fibroblasts were washed three times with ice-cold PBS and harvested by scraping in lysis buffer without $1 \%$ NP-40. Protein concentration in the sonicated lysates was determined using the Bradford assay and adjusted with lysis buffer lacking $1 \% \mathrm{NP}-40$ to a concentration of $1 \mathrm{mg} / \mathrm{ml}$. Then, $900 \mu \mathrm{g}$ of total protein was used for ultracentrifugation. The supernatant was transferred to another tube, and NP40 was added (end concentration 1\% NP-40). The pellet was dissolved in lysis buffer with 1\% NP-40 and sonicated. The supernatant (soluble) and pellet (membrane) fractions were then incubated with GST-RBD or GST-PAK, as described above. These experiments were performed in triplicate.

\section{Immunoblot analysis}

To allow comparisons between control and MKD cells, protein fractions of the two cell lines from one experiment were separated on the same 12\% SDS-polyacrylic acid (PAA) gel and subsequently transferred onto nitrocellulose membranes by semidry blotting. To verify equal transfer of proteins, each blot was reversibly stained with Ponceau S. prior to incubation with antibodies. Membranes were then incubated with RhoA monoclonal antibody (sc-418; Santa Cruz Biotechnology, Santa Cruz, CA, USA) diluted 1:1,000, Rac1 monoclonal antibody (Upstate Biotechnolo- gy, Lake Placid, NY, USA) diluted 1:10,000 or, to verify for equal amounts of input protein, $\beta$-actin monoclonal antibody (Sigma-Aldrich, St. Louis, MO, USA) diluted 1:10,000. Antigen-antibody complexes were visualized with rabbit anti-mouse horseradish peroxidase conjugate (DAKO, Glostrup, Denmark) using the enhanced chemiluminescence system (Amersham Biosciences, Little Chalfont, UK). Densitometric analysis of the immunoblots (same exposure for both cell lines) was performed using Advanced Image Data Analyzer (AIDA) software (Raytest, Strauenhardt, Germany).

\section{Statistical analysis}

Statistical analysis was performed using one-way analysis of variance (ANOVA) followed by Dunnett's post hoc test or paired Student's $t$ test. $P$ values less than 0.05 were considered significant.

\section{Results}

Effect of simvastatin on RhoA and Rac1 localization and activation

Previously, we showed that under standard culturing conditions, fibroblasts from MKD patients have similar levels of geranylgeranylated RhoA in their membranes, as observed in control cells, despite MK deficiency. Inhibition of HMG-CoA reductase, however, resulted in a stronger decrease of membrane-bound (i.e., geranylgeranylated) RhoA in MKD cells than in control cells (Houten et al 2003b). Using the same approach, i.e., reducing the flux through the pathway by incubating control and MKD cells with simvastatin, an inhibitor of HMG-CoA reductase, we now studied the effect on localization and activation of RhoA and Rac1. When control and MKD cells were cultured in the absence of simvastatin followed by separation into membrane and soluble fractions, we observed somewhat higher levels of membrane-bound RhoA in MKD cells than in control cells (Fig. 1b, immunoblots), whereas membrane-bound Rac1 levels were slightly lower in MKD cells than in control cells (Fig.1g, immunoblots). Incubation with simvastatin decreased the levels of membrane-bound RhoA in both control and MKD cells (Fig. 1b). However, MKD cells were more sensitive to treatment with simvastatin, already resulting in decreased levels of membrane-bound RhoA after incubation with $0.02 \mu \mathrm{M}$ simvastatin. The levels of RhoA protein in soluble fractions increased in both control and MKD cells (Fig. 1c). Although the relative increase of soluble RhoA was higher in control cells after simvastatin treatment, levels of soluble RhoA were higher in MKD cells, which 
A. Input

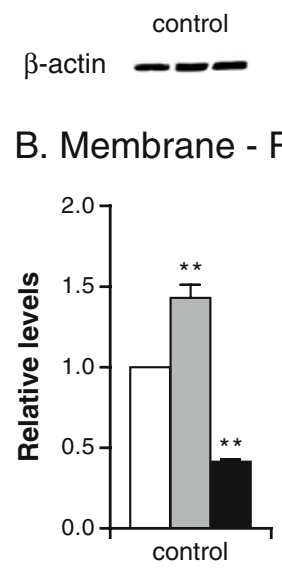

RhoA
MKD

$\beta$-actin
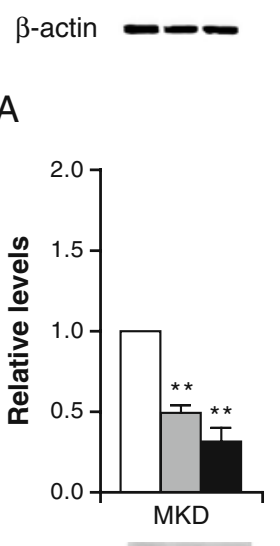

RhoA

\section{F. Input}

$\beta$-actin

G. Membrane - Rac1

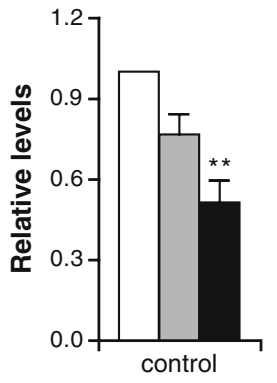

Rac1

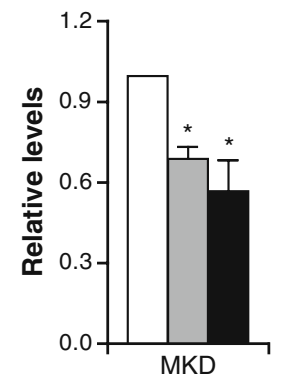

Rac1

\section{Soluble - RhoA}

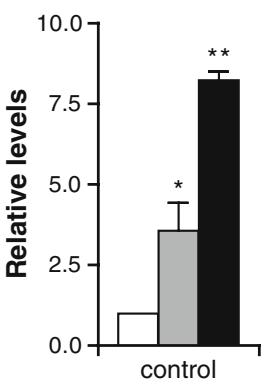

RhoA

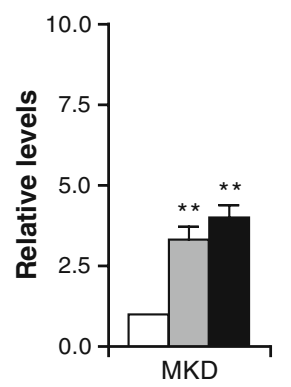

RhoA
H. Soluble - Rac1

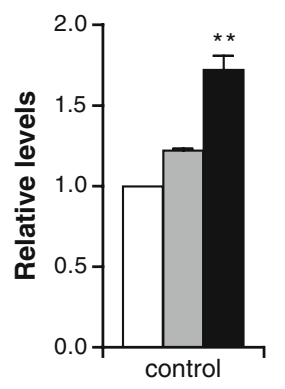

Rac1

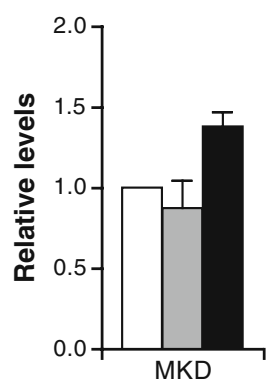

Rac1
D. Input

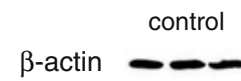

E. Active - RhoA

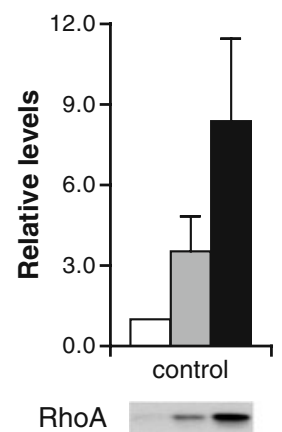

RhoA control

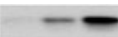

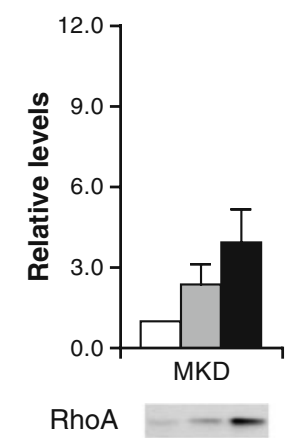

I. Input
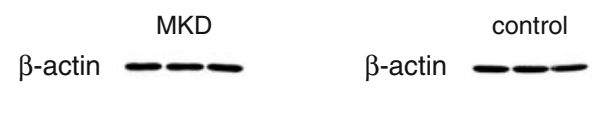

J. Active - Rac1
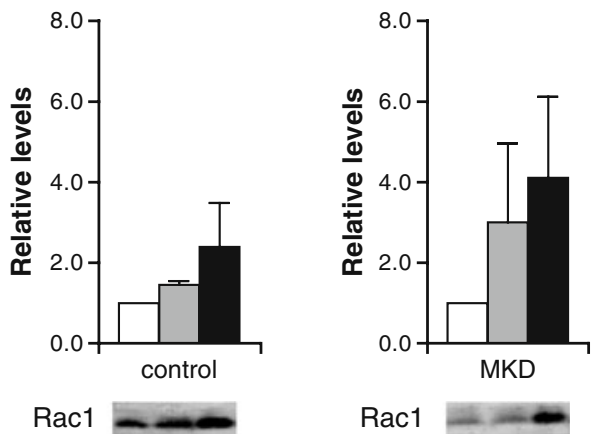

Rac1

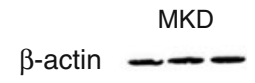

$\square 0 \mu \mathrm{M}$ simvastatin

$0.02 \mu \mathrm{M}$ simvastatin

$0.2 \mu \mathrm{M}$ simvastatin

Fig. 1 Effect of simvastatin on the localization and activation of RhoA (a-e) and Rac1 (f-j). Control and mevalonate kinase deficient (MKD) cells were incubated with 0 (white bar; set as 100\%), 0.02 (grey bar), or $0.2 \mu \mathrm{M}$ (black bar) simvastatin for 2 days. Equal amounts of cell lysate $(\mathbf{a}, \mathbf{f})$ were analyzed for relative levels of RhoA (b) and Rac1 (g) associated with the membranes and relative soluble levels of RhoA (c) and Rac1 (h). In separate experiments, equal amounts of cell lysate (d, i) were analyzed for relative levels of active RhoA (e) and Rac1 (j). Bars show the mean and standard error of the mean (SEM) of three independent experiments. Immunoblots show the results of one representative experiment. Statistic analysis of observed effects of treatments was performed with one-way analysis of variance followed by Dunnett's post hoc test. $*=P<0.05$, ** $=$ $P<0.01$ 
can be explained by the fact that untreated MKD cells already have higher levels of soluble RhoA.

Levels of membrane-bound Rac1 also decreased upon incubation with increasing simvastatin concentrations, paralleled with increasing levels of soluble Rac1 (Fig. 1g, h). However, with respect to Rac1 localization, MKD cells do not appear to be more sensitive toward inhibition with simvastatin than do control cells. Overall, the effects of simvastatin treatment were more pronounced for RhoA than for Rac1. Incubation with simvastatin led to an increase in the total levels of active, GTP-bound RhoA and Rac1 in both control and MKD cells. The relative increase in active RhoA was larger than the relative increase in active Rac1 (Fig. 1e, j).

The effect of GGTI on RhoA and Rac1 localization and activation

Earlier studies indicated that the effect of simvastatin on the localization of RhoA is due to a shortage of geranylgeranyl diphosphate (GGPP) and can be prevented by the addition of geranylgeraniol (GGOH) (Houten et al 2003b). To confirm that the observed effect of simvastatin on RhoA and Rac1 activation was due to a shortage of GGPP, we studied the effect of GGTI, an inhibitor of geranylgeranyl transferase, on localization and activation of RhoA and Rac 1 and found that GGTI had a similar effect as simvastatin. Incubation with GGTI led to a reduction in membrane-bound RhoA and Rac1 that was similar for both control and MKD cells (Fig. 2b, g) and, in parallel, an increase in the levels of soluble RhoA and Rac1 (Fig. 2c and 2h).

As observed with simvastatin, inhibition by GGTI also led to activation of total RhoA in both control and MKD cells (Fig. 2e). After treatment with GGTI, Rac1 was also activated in MKD cells; however, no Rac1 activation was detected in control cells (Fig. 2j). Thus, the effect of specific inhibition of geranylgeranylation by GGTI was more pronounced for RhoA than Rac1, which is comparable with results obtained after simvastatin treatment.

Effect of simvastatin on activation of soluble and membrane-bound RhoA and Rac1

To determine whether the observed increase of active GTPbound RhoA and Rac1 during the different culturing conditions was due to activation of soluble or membranebound RhoA and Rac1, we studied separately the effect of simvastatin. When MKD and control cells were cultured in the absence of simvastatin, levels of active RhoA protein in the soluble fraction were markedly higher in MKD cells, whereas levels of active membrane-bound RhoA were similar in both control and MKD cells (Fig. 3b, c). Incubation with simvastatin led to an increase of active soluble RhoA and a decrease of active membrane-bound RhoA in a concentration-dependent manner in both control and MKD cells. Again, MKD cells were more sensitive to treatment with simvastatin, with markedly increased levels of active soluble RhoA and decreased levels of active membrane-bound RhoA already observed after incubation with low concentrations of simvastatin.

In contrast to active RhoA, levels of active Rac1 protein in soluble and membrane fractions were similar in control and MKD cells in the absence of simvastatin (Fig. 3e, f). In the presence of simvastatin, there was a decrease in active membrane-bound Rac1 and an increase in active soluble Rac1, with MKD cells being more sensitive to lower concentrations of simvastatin than were control cells.

\section{Discussion}

Although the MK enzyme activity can barely be detected in MKD cells, we previously showed that protein isoprenylation is usually rather normal when these cells are cultured under normal conditions (Houten et al 2003b). This is due to increased activity of HMG-CoA reductase, which leads to elevated levels of mevalonate and a virtually normal flux through the isoprenoid biosynthesis pathway (Gibson et al 1990; Hoffmann et al 1997; Houten et al 2003b). Because MKD fibroblasts depend on elevated levels of mevalonate to maintain the flux through the pathway, they are more sensitive to simvastatin, an inhibitor of HMG-CoA reductase, which is the enzyme producing mevalonate (Houten et al 2003b). Indeed, in MKD fibroblasts, a low concentration of simvastatin already inhibits the pathway, resulting in a substantial reduction of membrane-bound RhoA. Although the relative decrease of membrane-bound Rac1 is similar for control and MKD cells, we also observed less membrane-bound Rac1 in MKD cells after incubation with simvastatin. However, the effect of inhibiting the isoprenoid biosynthesis pathway on isoprenylation of Racl is less pronounced than for RhoA, which may be due to different turnover rates of these two proteins. Because small GTPases require the isoprenyl moiety to translocate to the membrane to allow binding to their effectors, inhibition of the isoprenoid biosynthesis pathway is expected to disturb proper functioning of such small GTPases. Indeed, we believe our observations for RhoA and Rac1 will also pertain to other proteins that rely on geranylgeranylation, including other members of the small GTPase family.

The observed effect of simvastatin on protein isoprenylation of RhoA and Racl is due to a shortage of geranylgeranyl pyrophosphate, and it was previously shown that this shortage can be replenished by the addition of mevalonate or GGOH (Houten et al 2003b). Our experiments with GGTI confirm that increased activation of 
Fig. 2 Effect of geranylgeranyltransferase inhibitor (GGTI) on the localization and activation of RhoA (a-e) and Rac1 (f-j). Control and mevalonate kinase deficient (MKD) cells were incubated with $0 \mu \mathrm{M}$ (white bar; set as $100 \%$ ) or $20 \mu \mathrm{M}$ (black bar) GGTI for 2 days. Equal amounts of cell lysate $(\mathbf{a}, \mathbf{f})$ were analyzed for relative levels of RhoA (b) and Rac1 (g) associated with membranes and relative soluble levels of RhoA (c) and Rac1 (h). In separate experiments, equal amounts of cell lysate $(\mathbf{d}, \mathbf{i})$ were analyzed for relative levels of active RhoA (e) and Rac1 (j). Bars show the mean and standard error of the mean (SEM) of three independent experiments. Immunoblots show the results of one representative experiment. Statistic analysis of observed effects of treatments was performed with paired Student's $t$ test; $*=P<0.05, * *=P<0.01$
A. Input

$\beta$-actin $\stackrel{\text { control }}{-} \quad \beta$-actin -

B. Membrane - RhoA

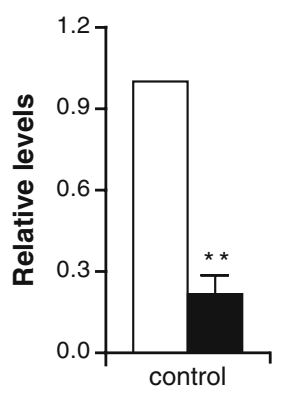

RhoA

C. Soluble - RhoA

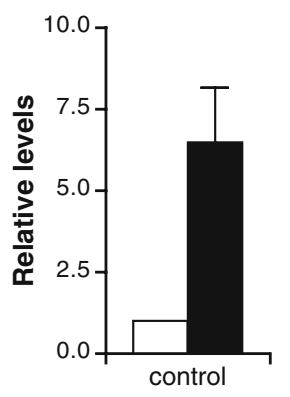

RhoA

D. Input

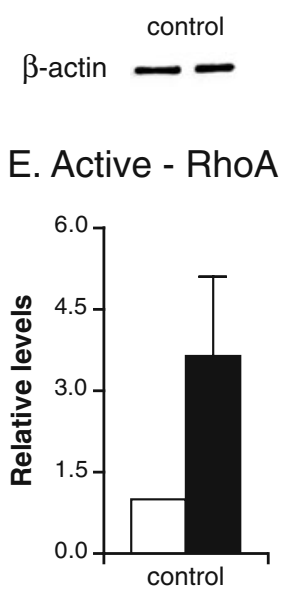

RhoA

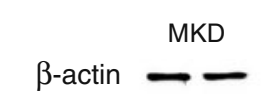

RhoA

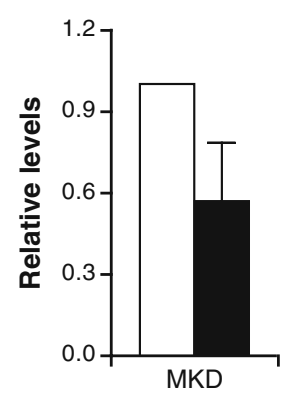

RhoA - -
F. Input $\beta$-actin - control $\quad \beta$-actin $=$
G. Membrane - Rac1

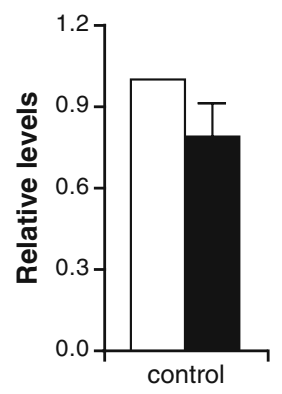

Rac1

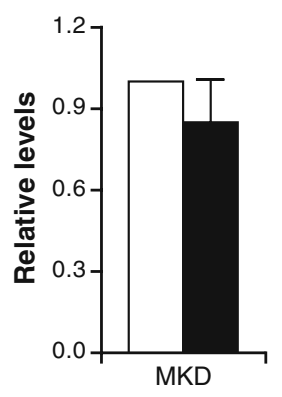

Rac1
H. Soluble - Rac1
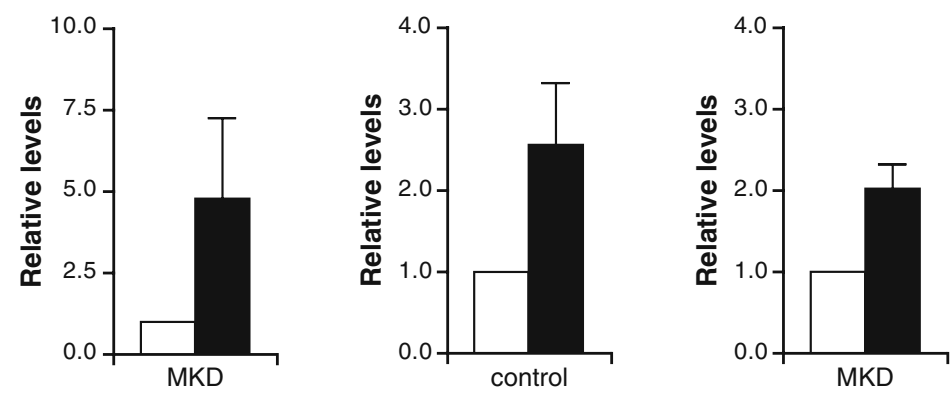

Rac1

Rac1

I. Input

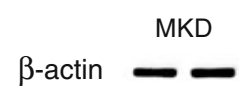

\section{J. Active - Rac1}

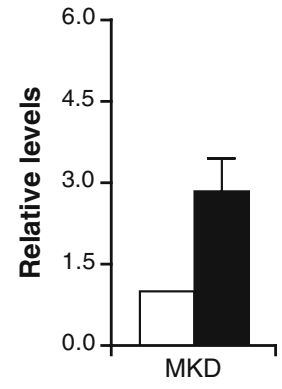

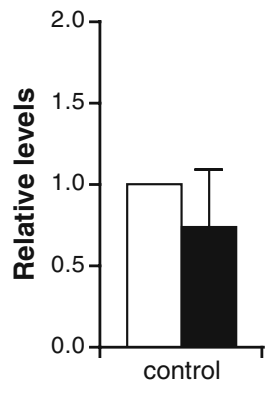

Rac1

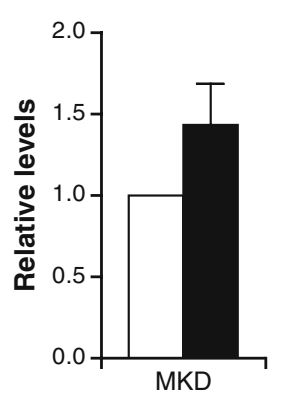

Rac1
RhoA and Rac1 by simvastatin is also the result of a shortage of geranylgeranyl moieties. The increase in total cellular levels of active RhoA and Rac1 due to simvastatin appears primarily, if not solely, caused by activation of soluble RhoA and Rac1 and not membrane-bound RhoA and Rac1. A possible explanation for this could be the difference in the ability of isoprenylated and nonisoprenylated proteins to interact with regulatory proteins. Because 
Fig. 3 Effect of simvastatin on activation of membrane-bound and soluble RhoA (a-c) and Rac1 (d-f). Control and mevalonate kinase deficient (MKD) cells were incubated with 0 (white bar), 0.02 (grey bar), or $0.2 \mu \mathrm{M}$ (black bar) simvastatin for 2 days. Equal amounts of cell lysate (a, d) were analyzed for relative levels of active RhoA (b) and active Rac1 (e) associated with the membranes (white bar set as 100\%) and relative soluble levels of active RhoA (c) and active Rac1 (f) (black bar set as $100 \%$ ). Bars show the mean and standard error of the mean (SEM) of three independent experiments. Immunoblots show the results of one representative experiment. $n d$ not detected. Statistical analysis of observed treatments effects was performed with oneway analysis of variance followed by Dunnett's post hoc test; $*=P<0.05, * *=P<0.01$
A. Input

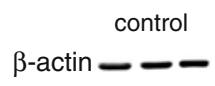

B. Membrane - active RhoA

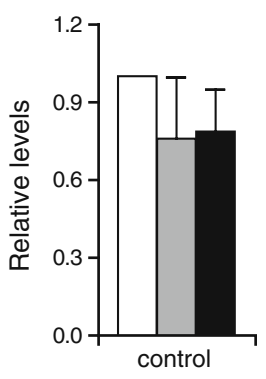

RhoA

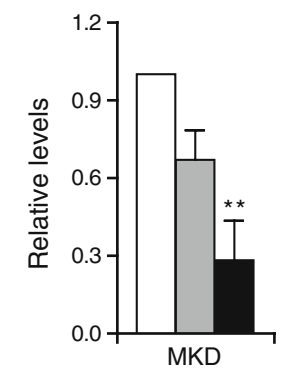

RhoA
D. Input

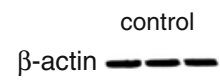

E. Membrane - active Rac1

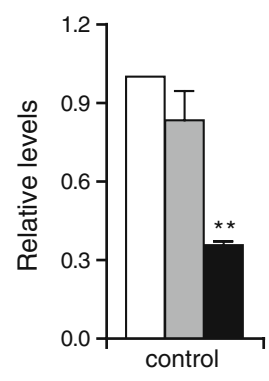

Rac1

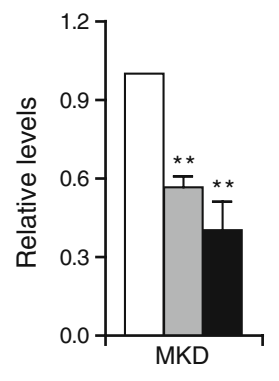

Rac1

\section{Soluble - active RhoA}

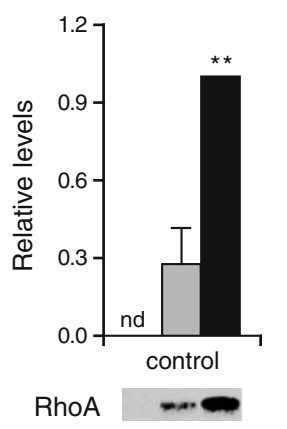

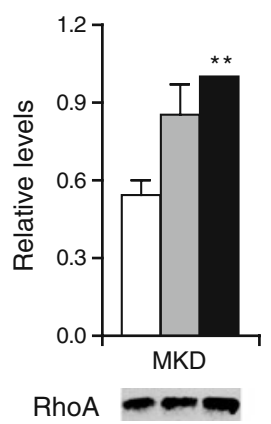

F. Soluble - active Rac1
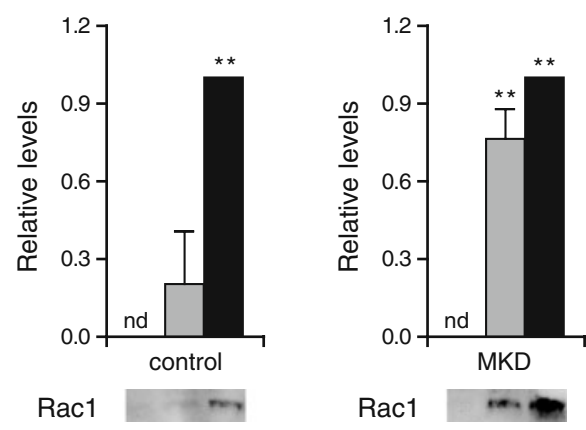

$0 \mu \mathrm{M}$ simvastatin

$0.02 \mu \mathrm{M}$ simvastatin

$0.2 \mu \mathrm{M}$ simvastatin

the isoprenyl moiety plays a critical role in interaction with Rho-GDI (Bustelo et al 2007), nonisoprenylated GTPases will no longer be inhibited by Rho-GDI and, consequently, intrinsic nucleotide exchange, i.e., GDP release and GTP binding, may occur. Moreover, it has been shown that nonisoprenylated Racl and RhoA have no or a much weaker interaction with GEFs (Ando et al 1992; Medley et al 2000) and GAPs (Molnar et al 2001) than the isoprenylated forms, implying that activation and inactivation of nonisoprenylated GTPases is compromised. Because intracellular GTP levels are higher than GDP levels, binding to GTP will be preferred over GDP (Carlucci et al 1997). Therefore, nonisoprenylated GTPases are likely to accumulate in the GTP-bound, active form.

Previously, we showed in vitro that treatment of MKD cells with simvastatin may lead to an increase of residual MK activity, whereas at the same time, the consequence of inhibiting $\mathrm{HMG}-\mathrm{CoA}$ reductase appears to be negative for flux through the isoprenoid biosynthesis pathway (Schneiders et al 2006). These findings indicated that one needs to be cautious when treating MKD patients with simvastatin, because the balance between inhibiting HMGCoA reductase and inducing $\mathrm{MK}$ activity may be critical, especially in MKD patients in whom the pathway flux is very sensitive to external influences. This was also suggested by the negative outcome of treating two patients with severe MKD-i.e. classic mevalonic aciduria-with lovastatin, a drug that is similar to simvastatin (Hoffmann 1993). This treatment provoked severe clinical crises in those patients. In contrast, treatment of six patients with a milder MKD - i.e. hyper-IgD syndrome-with simvastatin (Simon et al 2004) did not provoke clinical crises. Although no statistical difference was observed with respect to the severity, frequency, and occurrence of febrile attacks, a decrease in the total number of febrile days was observed. These findings are consistent with this study and our previous observations that, in vitro, cells from patients with mevalonic aciduria appear more sensitive to simvastatin compared with cells from patients with hyper-IgD syndrome or those from control individuals (Houten et al 2003b).

In summary, we showed that protein isoprenylation in MKD cells is more sensitive to inhibition by simvastatin than in control cells, resulting in an increased, ectopic activation of soluble RhoA and Racl in MKD cells. We hypothesize that such incorrect (ectopic) subcellular local- 
ization of activated small GTPases leads to inappropriate intracellular signaling, which may contribute to the inflammatory phenotype observed in MKD. This may be due to a failure to induce certain signaling pathways or incorrect induction of other signaling pathways involved in the up- or downregulation of inflammation, or both. In future studies, the effect of the disturbed, ectopic activation of these and other GTPases on their downstream targets will be studied, which may provide important insights into the pathophysiology underlying the inflammatory episodes observed in MKD patients.

Acknowledgements We thank Drs. Sander Houten and Ronald Wanders for their valuable input and critical discussions throughout this study. This research was supported by grant 912-03-024 of ZonMW.

Open Access This article is distributed under the terms of the Creative Commons Attribution Noncommercial License which permits any noncommercial use, distribution, and reproduction in any medium, provided the original author(s) and source are credited.

\section{References}

Ando S, Kaibuchi K, Sasaki T et al (1992) Post-translational processing of rac $\mathrm{p} 21 \mathrm{~s}$ is important both for their interaction with the GDP/GTP exchange proteins and for their activation of NADPH oxidase. J Biol Chem 267:25709-25713

Bustelo XR, Sauzeau V, Berenjeno IM (2007) GTP-binding proteins of the Rho/Rac family: regulation, effectors and functions in vivo. Bioessays 29:356-370

Carlucci F, Rosi F, Di PC, Marinello E, Pizzichini M, Tabucchi A (1997) Purine nucleotide metabolism: specific aspects in chronic lymphocytic leukemia lymphocytes. Biochim Biophys Acta 1360:203-210

Casey PJ, Seabra MC (1996) Protein prenyltransferases. J Biol Chem 271:5289-5292

Frenkel J, Rijkers GT, Mandey SH et al (2002) Lack of isoprenoid products raises ex vivo interleukin-1beta secretion in hyperimmunoglobulinemia D and periodic fever syndrome. Arthritis Rheum 46:2794-2803

Gibson KM, Hoffmann G, Schwall A et al (1990) 3-Hydroxy-3methylglutaryl coenzyme A reductase activity in cultured fibroblasts from patients with mevalonate kinase deficiency: differential response to lipid supplied by fetal bovine serum in tissue culture medium. J Lipid Res 31:515-521
Hoffmann GF, Charpentier C, Mayatepek E et al (1993) Clinical and biochemical phenotype in 11 patients with mevalonic aciduria. Pediatrics 91(5):915-21

Hoffmann GF, Wiesmann UN, Brendel S, Keller RK, Gibson KM (1997) Regulatory adaptation of isoprenoid biosynthesis and the LDL receptor pathway in fibroblasts from patients with mevalonate kinase deficiency. Pediatr Res 41:541-546

Houten SM, Frenkel J, Rijkers GT, Wanders RJ, Kuis W, Waterham HR (2002) Temperature dependence of mutant mevalonate kinase activity as a pathogenic factor in hyper-IgD and periodic fever syndrome. Hum Mol Genet 11:3115-3124

Houten SM, Frenkel J, Waterham HR (2003a) Isoprenoid biosynthesis in hereditary periodic fever syndromes and inflammation. Cell Mol Life Sci 60:1118-1134

Houten SM, Schneiders MS, Wanders RJ, Waterham HR (2003b) Regulation of isoprenoid/cholesterol biosynthesis in cells from mevalonate kinase-deficient patients. J Biol Chem 278:57365743

Mandey SH, Kuijk LM, Frenkel J, Waterham HR (2006) A role for geranylgeranylation in interleukin-1beta secretion. Arthritis Rheum 54:3690-3695

McTaggart SJ (2006) Isoprenylated proteins. Cell Mol Life Sci 63:255-267

Medley QG, Serra-Pages C, Iannotti E et al (2000) The trio guanine nucleotide exchange factor is a RhoA target Binding of RhoA to the trio immunoglobulin-like domain. J Biol Chem 275:3611636123

Molnar G, Dagher MC, Geiszt M, Settleman J, Ligeti E (2001) Role of prenylation in the interaction of Rho-family small GTPases with GTPase activating proteins. Biochemistry 40:1054210549

Reid T, Furuyashiki T, Ishizaki T et al (1996) Rhotekin, a new putative target for Rho bearing homology to a serine/threonine kinase, $\mathrm{PKN}$, and rhophilin in the rho-binding domain. $\mathrm{J}$ Biol Chem 271:13556-13560

Sander EE, van Delft S, ten Klooster JP et al (1998) Matrix-dependent Tiam1/Rac signaling in epithelial cells promotes either cell-cell adhesion or cell migration and is regulated by phosphatidylinositol 3-kinase. J Cell Biol 143:1385-1398

Schneiders MS, Houten SM, Turkenburg M, Wanders RJ, Waterham HR (2006) Manipulation of isoprenoid biosynthesis as a possible therapeutic option in mevalonate kinase deficiency. Arthritis Rheum 54:2306-2313

Simon A, Drewe E, van der Meer JW et al (2004) Simvastatin treatment for inflammatory attacks of the hyperimmunoglobulinemia D and periodic fever syndrome. Clin Pharmacol Ther $75: 476-483$

Takai Y, Sasaki T, Matozaki T (2001) Small GTP-binding proteins. Physiol Rev 81:153-208

Waterham HR (2006) Defects of cholesterol biosynthesis. FEBS Lett 580:5442-5449 